\title{
ANEURISMAS INTRACRANIANOS MÚLTIPLOS
}

\author{
JOSE CARLOS LYNCH* - RICARDO ALVES DE ANDRADE**
}

\begin{abstract}
RESUMO - Os aneurismas múltiplos correspondem de 15 a $31 \%$ dos aneurismas cerebrais. Em nosso material encontramos $19,4 \%$ de aneurismas múltiplos. Trinta e oito pacientes foram incluídos neste estudo e totalizaram 89 aneurismas e 4 infundíbulos. $\mathrm{Em} 71,0 \%$ dos casos foram encontrados 2 aneurismas; em 18,4\%, 3 aneurismas; e em 10,4\%, 4 ou 5 aneurismas. As artérias carótida, comunicante anterior, comunicante posterior e cerebral média foram os sítios mais frequentes dessas lesões. Observamos nitido predomínio do sexo feminino (2,45:1). A média de idade foi 47 anos, com os extremos variando entre 16 e 72 anos. A hemorragia subaracnóidea foi a mais comum das formas de expressão clínica (86,8\%). A mortalidade cirúrgica nesta série foi de 3,5\%, o que nos leva a concluir que pacientes com aneurismas múltiplos devem ser submetidos a tratamento microcirúrgico para isolar da circulação os aneurismas que, rompendo, podem colocar em risco a vida dos pacientes.
\end{abstract}

PALAVRAS CHAVE: aneurisma intracraniano, aneurismas múltiplos, cirurgia.

\section{Multiple intracranial aneurysms.}

SUMMARY - Thirty eight patients with multiple intracranial aneurysms were studied. They correspond to $19.4 \%$ of all aneurysms treated over a twelve year period in the Servidores do Estado Hospital. 89 aneurysms and 4 infundibuli were detected. In $71.0 \%$ of the patients, two aneurysms were found; in $18.4 \%$, three aneurysms; and in $10.4 \%, 4$ or 5 aneurysms were observed. Twenty-seven patients were women and $11 \mathrm{men}$, ranging in age from 16 to 72 (average 47 years old). Subarachnoid hemorrhage was found in 36 patients (86.8\%).' The operative mortality in this series was $3.5 \%$. We concluded that patients with multiple intracranial aneurysms should have all aneurysms, that can bleed, clipped through standard micro-neurosurgery technics.

KEY WORDS: intracranial aneurysm, multiple aneurysms, surgery.

Bigelow 4 , em 1955, reviu pela primeira vez todo o material relativo a aneurismas cerebrais múltiplos publicados na literatura mundial. Nessa análise de 2.237 casos de aneurismas intracranianos, ele detectou aproximadamente $10 \%$ de aneurismas múltiplos. No estudo cooperativo de aneurismas intracranianos e hemorragia subaracnóidea (HSA) foram detectadas lesões múltiplas em $18,5 \%$ dos casos estudados por angiografia e $22 \%$ nos estudados por necropsia 15 . Hoje aceita-se que os aneurismas cerebrais múltiplos correspondam de 15 a $31 \%$ dos casos de aneurismas intracranianos 28 .

O objetivo deste estudo é resumir nossa experiência com um grupo de 38 pacientes com aneurismas cerebrais múltiplos e tentar determinar a melhor forma de se tratar pacientes com a condição.

\section{MATERIAL E MÉTODO}

No período compreendido entre 1976 e 1988, tivemos a oportunidade de tratar 221 pacientes com aneurisma cerebral. Destes, 43 apresentavam aneurismas múltiplos, corres-

Serviço de Neurocirurgia do Hospital dos Servidores do Estado, RJ: * Chefe de Serviço; ** Chefe da Clínica.

Dr. Jose Carlos Lynch - Rua Jardim Botanico 700/318 - 22461 Rio de Janeiro RJ - Brasil 
pondendo a $19,45 \%$ dos casos. Dos 43 pacientes com lesões múltiplas, 5 foram excluidos, por não apresentarem informações completas. Os 38 pacientes incluídos neste estudo estão relacionados na Tabela 1 , em ordem cronológica de atendimento. Os prontuários e angiografias foram revistos e os dados relacionados com idade, sexo, sintomas, grau de comprometimento neurológico, achados radiológicos, número, tamanho e localização dos aneurismas, tratamento e resultados, coletados (Tabela 1).

Nos 38 pacientes, encontramos 89 aneurismas e 4 infundíbulos, fazendo um total de 93 lesões vasculares. Vinte e sete pacientes apresentavam 2 aneurismas $(71,05 \%) ; 7$ pacientes eram portadores de 3 aneurismas $(18,42 \%) ; 2$ pacientes revelaram 4 aneurismas e em 2 pacientes encontramos 5 aneurismas $(5,26 \%)$. Estas lesões se distribuíram da seguinte maneira: na Artéria Vertebral (Vert), na Choroidea Anterior (ChA), na Pericalosa (Pcal) e na Cerebral Anterior (CAn), 1 aneurisma; na Basilar (Bas) e Oftálmica (Oft), 2 aneurismas na Artéria Carótida (Ca), 14 aneurismas; na Artéria Comunicante Anterior (CoA), 15 aneurismas; na Artéria Comunicante Posterior (CoP), 25 aneurismas e na Cerebral Média (CM), 31 aneurismas. Nos casos de aneurismas duplos encontramos as seguintes associaçóes: Artéria CA-CA, 8 casos; CM-CM, 4 casos; CoA-CA, 4 casos; CoA-CM, 3 casos; CA-CM, 2 casos; CA-Oft, 2 casos; CM-Pcal, CoA-Vert, CA-Bas, 1 caso. Observamos nitido predominio feminino: 27 mulheres e 11 homens. A média de idade dos pacientes foi 47,3 anos, com os extremos variando entre 16 e 72 anos. A década mais afetada foi a sexta.' Em 33 casos, o aneurisma se manifestou por HSA (86,8\%). Em 3, a apresentação foi de compressåo de nervo craniano (7,8\%); em um paciente, cefaléia sem HSA e, em outro, a associação de HSA e compressão do terceiro nervo craniano. Em 19 pacientes (50\%) detectamos hipertensão arterial sistêmica. Considerando todos os 74 aneurismas, o tamanho variou de 3 a $42 \mathrm{~mm}$.

LOCALIZAÇAO DO ANEURISMA ROTO - Dos 38 casos analisados, 4 faleceram, sem cirurgia (casos 10,23, 32, 38). Um paciente (caso 7) só teve cefaléia sem HSA e dois apresentaram aneurismas cavernosos bilaterais (casos 31 e 33). Três pacientes sangraram e foram operados, mas não se conseguiu determinar com certeza qual dos aneurismas havia rompido (casos 8, 25 e 34). Restaram, então, 28 pacientes que tiveram HSA e cujo aneurisma responsável pela hemorragia foi detectado pela cirurgia. Destes 28 pacientes, em $96 \%$ das vezes o aneurisma roto era o de maior tamanho; somente em um caso (18) o aneurisma menor tinha sido responsável pela HSA.

Em 7 pacientes observamos presença de aneurisma de forma irregular. Em 6 casos o aneurisma irregular era o responsável pelo sangramento, mas estes eram também os de maior tamanho.

Hematoma intracerebral ou cisternal, detectado pela TCC ou angiografia, sinalizou o aneurisma roto em 5 ocasiōes (casos 4, 11, 17, 19, 22). Vasoespasmo (casos 5, 15), infarto cerebral (caso 12, 30, 31) também foram de grande ajuda na localização do aneurisma sintomático.

TRATAMENTO - Todos os pacientes foram submetidos a craniotomia pterional e operados com técnica microcirúrgica após a segunda semana de sangramento. Uma única exceção foi o caso 8, levado à cirurgia no quinto dia depois do sangramento.

Nosso objetivo foi sempre excluir da circulação todos os aneurismas que poderiam, em caso de ruptura, colocar em risco a vida do paciente. Porém, isto não foi alcançado em 7 pacientes. $O$ caso 1 desenvolveu edema cerebral pre-operatório, que impediu a clipagem do segundo aneurisma. Os casos 3 e 6 recusaram a segunda cirurgia. Nos casos 12 e 14, o segundo tempo cirúrgico não foi considerado, devido a doença sistêmica grave (neoplasia maligna e insuficiência renal em programa de hemodiálise). Doís pacientes (casos 31 e 33) com aneurismas cavernosos bilaterais, não foram submetidos a qualquer tratamento cirúrgico. Para melhor avaliação do tratamento nesta série, os pacientes foram divididos em trés grupos distintos: - Grupo I: aneurismas localizados em um mesmo hemisfério cerebral, abordados através de uma única cirurgia (casos 4, 8, 11, 14, 22, 24, 26, 37); - Grupo II: aneurismas localizados em lados diferentes: (A) operados através de duas cirurgias distintas (casos $5,13,15,16,17,35$ ) ou (B) operados por uma única cirurgia (com acesso contralateral) (casos 9, 18, 19, 20, 25, 27, 28, 30, 36); - Grupo III: aneurismas supra e infratentorial: (A) operado através de duas cirurgias distintas (caso 21) ou (B) operado por uma única cirurgia (caso 34).

\section{RESULTADOS}

A mortalidade geral foi de 23,6\%, ou seja, 9 pacientes. Destes 9 pacientes, 4 ressangraram e faleceram antes de serem submetidos a tratamento cirúrgico (casos 10, 23, 32, 38). Dois pacientes (casos 31 e 33) năo foram operados. 
Tabela 1 - Aneurismas múltiplos.

\begin{tabular}{|c|c|c|c|c|c|c|}
\hline Caso & $\begin{array}{l}\text { Idade } \\
\text { /Sexo }\end{array}$ & Sintromas & Grau & $\begin{array}{l}\text { Achados } \\
\text { radiológicos }\end{array}$ & Tratamento & Resultade \\
\hline 1 & $72 / F$ & HSA & IV & ACad, ACMD & Clip ACaD & $\begin{array}{l}\text { óbito: } 60 \text { dias } \\
\text { após a cirurgia }\end{array}$ \\
\hline 2 & $52 / \mathbf{M}$ & HSA & $\mathbf{I}$ & $\mathrm{ACoA}, \mathrm{ACaD}$ & Clip ACoA & Excelente \\
\hline 3 & $55 / F$ & HSA & II & $\mathrm{ACaE}, \mathrm{ACaD}$ & Clip ACaE & $\begin{array}{l}\text { Bom: hemipa- } \\
\text { resia discreta }\end{array}$ \\
\hline 4 & $52 / F$ & HSA & IV & $\mathrm{ACOA}, \mathrm{ACME}$ & $\begin{array}{l}\text { Clip ACoA } \\
\text { Clip ACME } \\
\text { Único tempo }\end{array}$ & $\begin{array}{l}\text { Vaso-espasmo } \\
\text { Obito: } 42 \text { dias } \\
\text { após a cirurgia }\end{array}$ \\
\hline 5 & $42 / F$ & HSA & II & ACMD, APcal & $\begin{array}{l}\text { Clip ACMD } \\
\text { Clip APcal } \\
\text { Dois tempoz }\end{array}$ & $\begin{array}{l}\text { Excelente } \\
\text { Torneiras }\end{array}$ \\
\hline $\begin{array}{l}6 \\
7\end{array}$ & $\begin{array}{l}52 / F \\
34 / F\end{array}$ & $\begin{array}{l}\text { HSA } \\
\text { Cefaleia }\end{array}$ & $\begin{array}{l}\text { II } \\
\text { I }\end{array}$ & $\begin{array}{l}\text { ACME, ACMD } \\
\text { ACaE, ACaD }\end{array}$ & $\begin{array}{l}\text { Clip ACME: } \\
\text { Exploração } \\
\text { ACaE LIG } \\
\text { CaD }\end{array}$ & $\begin{array}{l}\text { Excelente } \\
\text { Excelente }\end{array}$ \\
\hline 8 & $23 / M$ & HSA & I & $\mathrm{ACME}, \mathrm{ACoA}$ & $\begin{array}{l}\text { Clip ACME } \\
\text { Clip ACoA } \\
\text { Ứnico tempo }\end{array}$ & Excelente \\
\hline 9 & $52 / F$ & HSA & $\mathbf{I}$ & $\begin{array}{l}\text { ACaE } \\
\text { Inf. ACaD }\end{array}$ & $\begin{array}{l}\text { Clip ACaE } \\
\text { Exploração inf }\end{array}$ & Excelente \\
\hline 10 & $16 / F$ & HSA & I & $\begin{array}{l}\text { ACaD, ACaE } \\
\text { ACMD }\end{array}$ & - & $\begin{array}{l}\text { obito: ressangra- } \\
\text { mento antes da } \\
\text { cirurgia. }\end{array}$ \\
\hline 11 & $56 / F$ & HSA & IV & $\begin{array}{l}\text { Hidro inf } \\
\text { ACoA, 2ACME }\end{array}$ & $\begin{array}{l}\text { Shunt V-P } \\
\text { Clip ACoA } \\
\text { Clip 2ACME } \\
\text { Unico tempo }\end{array}$ & $\begin{array}{l}\text { óbito: } 67 \text { dias } \\
\text { após a cirurgia }\end{array}$ \\
\hline 12 & $51 / F$ & $\begin{array}{l}\text { HSA + } \\
\text { Compr. } \\
\text { 3० nervo }\end{array}$ & II & ACaE, ACaD & Clip ACaE & Excelente \\
\hline 13 & $52 / F$ & HSA & I & ACaE, ACaD & $\begin{array}{l}\text { Clip ACaE } \\
\text { Clip ACaD } \\
\text { Dois tempos }\end{array}$ & Excelente \\
\hline 14 & $25 / \mathbf{F}$ & HSA & II & $\begin{array}{l}\text { ACOA, } 2 A C M E \\
\text { ACMD }\end{array}$ & $\begin{array}{l}\text { Clip ACoA } \\
\text { Clip + Coag } \\
\text { 2ACME }\end{array}$ & Excelente \\
\hline 15 & $59 / F$ & HSA & III & $\begin{array}{l}\text { Hidro, ACoA } \\
\text { 2ACME, ACaD } \\
\text { ACMD }\end{array}$ & $\begin{array}{l}\text { Shunt VP } \\
\text { Clip ACoA } \\
\text { Clip 2ACME } \\
\text { Musc ACMD } \\
\text { Dois tempos }\end{array}$ & Excelente \\
\hline 16 & $39 / \mathrm{F}$ & HSA & II & ACMD, ACME & $\begin{array}{l}\text { Clip ACMD } \\
\text { Clip ACME } \\
\text { Dois tempos }\end{array}$ & $\begin{array}{l}\text { Infarto frontal } \\
\text { Excelente }\end{array}$ \\
\hline
\end{tabular}

ABas, artéria basilar; $A C a$, artéria carótida; $A C M$, artéria cerebral média; ACoa, artéria comunicante anterior; Aoft, artéria oftalmica; APcal, artéria pericalosa; Avert, artéria vertebral; bil, bilateral; cirurg cirurgia; coag, coagulaçáo; compr, compressão; D, direita; E, esquerda; F, feminino; $M$, masculino; HSA, hemorragia sub-aracnbidea; hidro, hidrocefalia; hemat, hematoma; inf, infundibulo; lig, ligadura; musc, muisculo; temp, temporal. 
Tabela 1 - Aneurismas múltiplos (continuação).

\begin{tabular}{|c|c|c|c|c|c|c|}
\hline Caso & $\begin{array}{l}\text { Idade } \\
\text { /Sexo }\end{array}$ & Sintomas & Grau & $\begin{array}{l}\text { Achados } \\
\text { radiológicos }\end{array}$ & Tratamento & Resultado \\
\hline 17 & $38 / F$ & HSA & IV & $\begin{array}{l}\text { Hidro } \\
\text { Hemat temp } \\
\text { ACMD, ACaE }\end{array}$ & $\begin{array}{l}\text { Shunt VP } \\
\text { Remoção hemat } \\
\text { Clip ACMD } \\
\text { Clip ACaE } \\
\text { Dois tempos }\end{array}$ & $\begin{array}{l}\text { Infarto temporal } \\
\text { Vegetativo }\end{array}$ \\
\hline 18 & $53 / F$ & $\mathrm{HSA} \times 2$ & I & $\begin{array}{l}\text { ACaE, ACoA } \\
\text { ACaD }\end{array}$ & $\begin{array}{l}\text { Musc + Gaze } \\
\text { ACaE, ACoA } \\
\text { ACaD } \\
\text { Único tempo }\end{array}$ & Excelente \\
\hline 19 & $50 / F$ & HSA & III & $\begin{array}{l}\text { Hemat frontal } \\
2 A C O A, A C M D\end{array}$ & $\begin{array}{l}\text { Clip 2ACOA } \\
\text { Clip ACMD } \\
\text { Ưnico tempo } \\
\text { Contralateral }\end{array}$ & Excelente \\
\hline 20 & $57 / F$ & $\mathbf{H S A} \times \mathbf{3}$ & II & $\begin{array}{l}\text { ACaE, ACaD } \\
\text { Oclusão CaD }\end{array}$ & $\begin{array}{l}\text { Musc + Gaze } \\
\text { ACaE + ACaD } \\
\text { Único tempo } \\
\text { Contralateral }\end{array}$ & Excelente \\
\hline 21 & $44 / F$ & HSA & I & ACoA, AVertE & $\begin{array}{l}\text { Clip ACoA } \\
\text { Clip Vert } \\
\text { Dois tempos }\end{array}$ & Excelente \\
\hline 22 & $32 / F$ & HSA & IV & $\begin{array}{l}\text { Hemat temp } \\
2 A C M\end{array}$ & $\begin{array}{l}\text { Remoção hemat } \\
\text { Clip + Coag } \\
2 \text { ACM }\end{array}$ & $\begin{array}{l}\text { Infarto temporal } \\
\text { Sepsis } \\
\text { obito: } 32 \text { dias } \\
\text { após a cirurgia }\end{array}$ \\
\hline 23 & $45 / M$ & HSA & II & $\begin{array}{l}\text { ABas } \\
\text { inf } \mathrm{CaD}\end{array}$ & - & $\begin{array}{l}\text { obito } \\
\text { Ressangrou }\end{array}$ \\
\hline 24 & $56 / \mathrm{M}$ & HSA & III & $2 \mathrm{ACaE}$ & Clip 2ACaE & Bom \\
\hline 25 & $67 / \mathrm{M}$ & $\mathrm{HSA} \times 2$ & IV & $\begin{array}{l}\text { ACoA, ACMD } \\
\text { ACME }\end{array}$ & $\begin{array}{l}\text { Clip ACoA } \\
\text { Clip ACMD } \\
\text { Clip ACME } \\
\text { Único tempo }\end{array}$ & $\begin{array}{l}\text { Insuf. resp. } \\
\text { obito: } 3 \text { dias } \\
\text { após a cirurgia }\end{array}$ \\
\hline 26 & $42 / F$ & HSA & I & $\mathrm{ACOA}, \mathrm{ACME}$ & $\begin{array}{l}\text { Clip ACoA } \\
\text { Clip ACME } \\
\text { Único tempo }\end{array}$ & Excelente \\
\hline 27 & $60 / F$ & HSA & I & $\begin{array}{l}\text { ACaE, 2ACME } \\
\text { inf }\end{array}$ & $\begin{array}{l}\text { Clip ACaE } \\
\text { Clip + Coag } \\
\text { 2ACME } \\
\text { Musc + Gaze } \\
\text { Inf CaD } \\
\text { Único tempo } \\
\text { Contralateral }\end{array}$ & Excelente \\
\hline 28 & $61 / M$ & HSA & 11 & ACaD, AOftE & $\begin{array}{l}\text { Clip AcaD } \\
\text { Musc + Gaze } \\
\text { AOftE } \\
\text { Único tempo } \\
\text { Contralateral }\end{array}$ & Excelente \\
\hline 29 & $36 / \mathrm{M}$ & HSA & $i$ & $\begin{array}{l}\text { AOftE } \\
\text { inf } \mathrm{CaD}\end{array}$ & $\begin{array}{l}\text { Clip OftE } \\
\text { Exploração inf } \\
\text { Único tempo } \\
\text { Contralateral }\end{array}$ & Excelente \\
\hline
\end{tabular}


Tabela 1 - Aneurismas múltiplos (continuasāo).

\begin{tabular}{|c|c|c|c|c|c|c|}
\hline Caso & $\begin{array}{l}\text { Idade } \\
\text { /Sexo }\end{array}$ & Sintomas & Grau & $\begin{array}{l}\text { Achados } \\
\text { radiológicos }\end{array}$ & Tratamento & Resultado \\
\hline 30 & $54 / F$ & $\begin{array}{l}\text { Compr. } \\
\text { 3o nervo E }\end{array}$ & I & $\begin{array}{l}\text { ACaE, ACoA } \\
\text { ACaD }\end{array}$ & $\begin{array}{l}\text { Clip ACaE } \\
\text { Clip ACoA } \\
\text { Clip ACaD } \\
\text { Unico tempo } \\
\text { Contralateral }\end{array}$ & $\begin{array}{l}\text { Infarto cápsula } \\
\text { interna e Hemi- } \\
\text { plegia }\end{array}$ \\
\hline 31 & $35 / F$ & $\begin{array}{l}\text { Compr. } \\
6^{\circ} \text { nervo } \mathbf{E} \\
5^{9} \text { nervo } \mathbf{E}\end{array}$ & III & $\mathrm{ACaD}, \mathrm{ACaE}$ & Conservador & Excelente \\
\hline 32 & $52 / F$ & HSA & III & $\mathrm{ACaE}, \mathrm{ACoA}$ & - & $\begin{array}{l}\text { óbito: ressangrou } \\
\text { antes da cirurg }\end{array}$ \\
\hline 33 & $52 / F$ & $\begin{array}{l}\text { Compr. } \\
6^{\circ} \text { nervo } \\
\text { Bil. }\end{array}$ & I & $\mathrm{ACaD}, \mathrm{ACaE}$ & Observação & Excelente \\
\hline 34 & $48 / F$ & HSA & II & $\begin{array}{l}\text { ACaD, ACaE } \\
\text { ACMD, ACaE } \\
\text { ABas }\end{array}$ & $\begin{array}{l}\text { Clip 5-AN. } \\
\text { Único tempo } \\
\text { Contralateral }\end{array}$ & Bom \\
\hline 35 & $37 / \mathbf{M}$ & HSA & I & ACMD, ACME & $\begin{array}{l}\text { Clip ACMD } \\
\text { Clip ACME } \\
\text { Dois tempos }\end{array}$ & Excelente \\
\hline 36 & $42 / M$ & HSA & I & ACaE, ACaD & $\begin{array}{l}\text { Clip ACaE } \\
\text { Coag ACaD } \\
\text { Contralateral }\end{array}$ & Excelente \\
\hline 37 & $53 / \mathrm{M}$ & HSA & I & ACoA, ACaD & $\begin{array}{l}\text { Clip ACoA } \\
\text { Coag ACaD }\end{array}$ & Excelente \\
\hline 38 & $36 / \mathbf{M}$ & HSA & 1 & ACMD, ACME & - & $\begin{array}{l}\text { óbito: ressangrou } \\
\text { antes da cirurg }\end{array}$ \\
\hline
\end{tabular}

A mortalidade cirúrgica, considerando-se até 30 dias após a cirurgia, foi de 3,5\%; somente um paciente morreu. Quatro pacientes vieram a falecer 32, 42, 60 e 67 dias após a cirurgia (casos $1,11,22,42)$.

Dois pacientes apresentaram mau resultado cirúrgico. No caso 17 , a paciente, vários meses após as cirurgias, permanece em coma superficial; ela foi operada em dois tempos e ocorreu oclusão de um ramo da artéria cerebral média e subsequente infarto cerebral; desenvolveu, também, hidrocefalia e se encontrava em grau IV, quando da segunda cirurgia. No caso 30, a paciente era grau I e está com hemiplegia, devido a infarto da cápsula interna direita. Vinte e um pacientes obtiveram excelentes resultados, voltaram a suas atividades anteriores, sem apresentar sequelas. Três pacientes tiveram bom resultado, ou seja, apesar de um discreto déficit motor, desenvolvem suas atividades habituais com desembaraco.

Todos os 24 excelentes ou bons resultados ocorreram em pacientes que, no momento da cirurgia, estavam em graus I a III da escala de Hunt e Hess.

No Grupo I, de um total de 8 casos, ocorreram 3 óbitos após 30 dias de cirurgia e obtivemos 5 excelentes ou bons resultados. No Grupo IIA, de um total de 6 casos, obtivemos 1 mau resultado o 5 excelentes resultados. No Grupo IIB, de um total de 9 casos, observamos 1 mau resultado, 1 obito e 8 excelentes resultados. No Grupo IIIA, de 1 caso, obtivemos 1 excelente resultado. No Grupo IIIB, havia 1 único paciente, com bom resultado. 


\section{COMENTARIOS}

Em nosso material, a incidência de aneurismas múltiplos, foi de $19,45 \%$ e este número é compatível ao relatado na literatura mundial 28 . Em nossa casuística, havia 27 mulheres; a bem conhecida maior frequência de aneurismas em mulheres, acentua-se no caso dos aneurismas múltiplos 5,15,16,21,32. Na literatura, o grupo etário mais afetado pela HSA em paciente com aneurismas múltiplos fica entre a quinta e a sexta décadas 5,9,11,16,19; nossa experiência também coincide com esses dados.

Nos 38 pacientes, observamos que: $71,05 \%$ apresentavam 2 lesões; 18,42\%, 3 lesões; $5,26 \%, 4$ lesões e 2 pacientes $(5,26 \%), 5$ aneurismas. Este padrão, com acentuado predomínio de lesões duplas, é uniformemente descrito na literatura $5,9,11,16,19$

Considerando somente os casos com lesões duplas, McKissock et al.16 encontraram as seguintes associações: CM-CM, 22\%; CM-CoA, 20\%: CM-CA, 12\%; CoA-CA, 8\%. Heiskanen 11 encontrou as seguintes: CM-CM, 24\%; CA-CA $21 \%$; Ca-CM, $12 \%$, $\mathrm{Coa}-\mathrm{CM}, 11 \%$; $\mathrm{CA}-\mathrm{CoA}, 10 \%$. No estudo cooperativo de hemorragia subaracnóidea e aneurismas intracranianos 15 é relatado que também as combinações de pares CA-CA, CM-CM são de maior incidência. Moyes 19 detectou em sua série: $C A+C A, 29 \%$; $\mathrm{CM}+\mathrm{CM}, 11 \% ; \mathrm{CA}+\mathrm{CoA}, 19 \% ; \mathrm{CoA}+\mathrm{CM}, 11 \%$. Em nosso material, os aneurismas localizados em ambas as carótidas foram os mais frequentes (8 casos), seguidos pelas associações de CA-CoA, CM-CM, CA-CM, CoA-CM. Encontramos, portanto, 24 aneurismas simétricos, os chamados em espelho.

Em todas as séries revistas, a HSA foi a forma de apresentação clínica majoritária dos aneurismas múltiplos, seguida pela compressão de nervos cranianos $5,9,10,11,16,19$. Em nossa experiência $86,8 \%$ dos casos manifestaram-se com HSA e somente $7,8 \%$, por compressão de nervos cranianos.

Considerando todos os seus segmentos, a artéria carótida foi o sítio onde encontramos o maior número de aneurismas (39 aneurismas, ou 40,6\%), seguido pela artéria cerebral média (31 aneurismas, ou 32,29\%) e pela comunicante anterior (15 aneurismas, ou 15,6\%). Hacker et al.9, reviram dados de 1201 aneurismas em 488 pacientes adultos com aneurismas múltiplos e localizaram a artéria carótida como originária dos aneurismas em $43 \%$ dos casos, seguida pela CM (27\%) e CoA (21\%). Este perfil é semelhante ao descrito por Mount e Brisman 18. Porém, McKissock et al.16 e Heiskanen 11 encontraram, em seus materiais, maior participação de aneurismas envolvendo a artéria cerebral média, secundados por aneurismas da carótida e da comunicante anterior.

Identificar o aneurisma que sangrou é parte importante do tratamento dos aneurismas múltiplos. Isto porque o aneurisma roto é o que, preferencialmente, coloca em risco a vida do paciente e, sempre que possível, deve ser o primeiro a ser tratado. Em seu trabalho clássico, Wood 32 mostrava que em $95 \%$ das vezes o aneurisma roto podia ser identificado e, em $87 \%$ das vezes, o aneurisma de maior tamanho havia sangrado. Em outra série a identificação foi de $77 \% 2$. Nehls et al.21 concluíram que a irregularidade do saco aneurismático é o fator mais importante na identificação do aneurisma roto. Em nosso material fomos capazes de confirmar o aneurisma roto, pela cirurgia, em 28 pacientes. Destes, em $96 \%$, o aneurisma roto era o de maior diâmetro. Em 7 pacientes observamos aneurisma irregular, em 6 casos eles tinham sido responsáveis pelo sangramento, sendo, também, os de maior tamanho.

O tratamento dos aneurismas múltiplos ainda levanta controvérsias e discussões. Em 1951, um paciente com dois aneurismas intracranianos foi submetido pela primeira vez a uma craniotomia para tratamento das duas lesões 3. Ainda na década de 50, Poppen e Fager 25, descrevem a primeira série de pacientes com aneurismas múltiplos diagnosticados em vida, tratados e acompanhados. Heiskanen e Marttila 12, Af Bjorkesten e Troup 1, McKissock et al.16, Paterson e Bond 24 defendiam a opinião de que somente o aneurisma que rompeu deveria ser objeto de tratamento cirúrgico. Anos mais tarde, Moyes 19, Salazar 26, Sanson et al.27, Drake e Girvin 7, Mount e Brisman 18 sustentaram que todos os aneurismas deveriam ser operados. Como argumento para isto, afirmaram que o risco de uma hemorragia subaracnóidea seria bem maior que o risco da segunda craniotomia. Jane et al.13, Moyes 19 e Sanson et al.27 acreditam que a tensão emocional, que surge no indivíduo que se sabe portador de um aneurisma cerebral, é fator importante a pesar na decisão de se operar um aneurisma intacto. Jane et al.13, estimam que um aneurisma íntegro tem $3 \%$ de chance de se romper a cada ano. Já para DuBoulay 8 , 0 risco anual de uma HSA 
fatal é de $5 \%$. Alguns autores 30 , preocupados em evitar uma craniotomia que poderia ser desnecessária, sugerem que os aneurismas assintomáticos, menores de $10 \mathrm{~mm}$, não devam ser operados. Segundo eles, estes aneurismas oferecem poucos riscos de ruptura. Ojemann 22 acredita que o limite de risco deva ser de $7 \mathrm{~mm}$. Crawford 6 observou que a maioria dos aneurismas se rompem entre 6 e $15 \mathrm{~mm}$. Kassel e Torner 14 detectaram que $71 \%$ dos aneurismas rotos possuíam mais de $10 \mathrm{~mm}$. No entanto, $13 \%$ dos aneurismas rotos eram menores que $5 \mathrm{~mm}$. Embora a maioria dos aneurismas se rompa quando o seu diâmetro externo atinge de 5 a $10 \mathrm{~mm}$, o comportamento biológico irregular destas lesões faz com que se torne impossível antecipar, com certeza, o momento de rompimento de um aneurisma cerebral. Em nosso material, 19 aneurismas rotos eram menores que $10 \mathrm{~mm}$ e um possuía $3 \mathrm{~mm}$ de diâmetro.

Wirth et al.31 reviram resultados coletados de várias séries publicadas sobre 260 pacientes com aneurismas intactos, operados com técnicas microcirúrgicas por diversos microcirurgiões. Neste grupo, a mortalidade encontrada foi zero e a morbidade foi $9 \%$. A decisão de se operar um aneurisma assintomático deve ser individualizada, baseando-se na idade do paciente, na sua condição clínica, no tamanho e localização do aneurisma e na experiência do cirurgião 5 . Se o aneurisma roto e o aneurisma assintomático estiverem localizados em um mesmo lado, a decisão fica facilitada porque ambos podem ser abordados por técnicas microcirúrgicas através de uma única craniotomia, em um único tempo. Em nossa casuística tivemos 8 casos nesta última situação. Não ocorreu qualquer óbito cirúrgico e obtivemos 4 excelentes cu bons resultados. Estes pacientes estavam nos graus I a III no momento da cirurgia. Neste grupo, ocorreram 3 óbitos tardios. Em todos estes casos os pacientes estavam no grau IV.

Quando um aneurisma é supratentorial e o segundo localizado no topo basilar, ou no segmento $\mathrm{P} 1$ da artéria cerebral posterior ou no segmento inicial da artéria cerebelosa superior, eles podem ser abordados através de uma única craniotomia pterional, dependendo da altura da clinóide posterior. Um único paciente, nesta situação, evoluiu bem (caso 34 ).

Quando os aneurismas estão situados em hemisférios opostos, usualmente se recomenda uma segunda craniotomia para tratar o aneurisma assintomático 18,19,26,27. Em nossa experiência isto aconteceu em 6 casos. Nenhum paciente faleceu. Obtivemos 5 excelentes resultados e 1 mau resultado.

Recentemente, alguns autores descreveram casos esporádicos em que uma única craniotomia pterional foi empregada para clipar aneurismas múltiplos, localizados em lados opostos 17,20,33,34. Porém, foram Oliveira et al.23 e Vadja et al.29 que sistematizaram e desenvolveram a técnica de se operar, através da microcirurgia, aneurismas múltiplos bilaterais em um único tempo cirúrgico. Tivemos a oportunidade de empregar esta técnica em 10 pacientes com aneurismas múltiplos bilaterais e ocorreram: 1 óbito, 1 mau resultado e 8 excelentes resultados.

$O$ acesso pterional contralateral é refinamento técnico que evita uma segunda cirurgia e diminui o tempo de permanência hospitalar. Porém, só deve ser utilizado em casos especiais, por neurocirurgiões com experiência em microcirurgia vascular cerebral e realizado em centros adequadamente estruturados para a moderna microcirurgia.

\section{REFERENCIAS}

1. Af Bjorkesten G, Troup H.' Multiple intracranial arterial aneurysms. Acta Chir Scand 1960, 118:387-391.

2. Almaani WS, Richardson AE. Multiple intracranial aneurysms: identifying the ruptured lesion. Surg Neurol 1978, 9:303-305.

3. Bassett RC. Multiple cerebral aneurysm: case report. J Neurosurg 1951, 8:132-134.

4. Bigelow NH. Multiple intracranial arterial aneurysms. Arch Neurol Psychiat 1955, 73:76-99.

5. Brisman R. Treatment of multiple and asymptomatic aneurysms. In Schmidek HH, Sweet WH (eds): Operative Neurosurgical Techniques, Indications, Methods and Results. Ed 2. Orlando: Grune \& Straton, 1988, p 1003-1007.

6. Crawford $T$. Some observations on the pathogenesis and natural history of intracranial aneurysms.' J Neurol Neurosurg Psychiat 1959, 22:259-266. 
7. Drake CF, Girvin JP. The surgical trelatment of subarachnoid hemorrhage with multiple aneurysm. In Morley TP (ed): Current Controversies in Neurosurgery. Philadelphia: Saunders, 1976, p 274-278.

8. DuBoulay GH. Some observations on the natural history intracranial aneurysms. Br J Radiol 1965, 38:721-757.

9. Hacker RJ, Krall JM, Fox JL. In Fox JL (ed): Intracranial Aneurysms. New York: Springer, 1983, Vol 1, p 19-62.

10. Hashimoto $N$, Handa $H$. The size of cerebral aneurysm in relation to repeated rupture. Surg Neurol 1983, 19:107-111.

11. Heiskanen $O$. Multiple intracranial arterial aneurysms. Acta Neurol Scand 1965, $41: 356-382$.

12. Heiskanen $O$, Marttila $I$. Risk of rupture of a second aneurysms in patients with multiple aneurysms J Neurosurg 1970, 32:295-298.

13. Jane JA, Winn HR, Richardson AE. The natural history of intracranial aneurysms: rebleeding rates during the acute and long term period and implication for surgical management. Clin Neurosurg 1976, 24:176-184.

14. Kassel NF, Torner JC. Size of intracranial aneurysms. Neurosurgery 1983, 3:291-297.

15. Locksley HB. Natural history of subarachnoid hemorrhage, intracranial aneurysms and arteriovenous malformations: based on 6368 cases in the cooperative study. In Saws AL, Paret GE, Locksley HB, Nishioka H (eds): Intracranial Aneurysms and Subarachnoid Hemorrhage, A Cooperative Study. Philadelphila: Lippincott, 1969, 37-108.

16. McKissock W, Richardson A, Walsh L, Owen E. Multiple intracranial aneurysms. Lancet 1964, 1:623-626.

17. Milenkovic Z, Gopic H, Antovic P, Jovicic V, Petrovic B. Contralateral pterional approach to a carotid ophthalmic aneurysm ruptured at surgery: case report. J Neurosurg 1982, $57: 823-825$.

18. Mount LA, Brisman R. Treatment of multiple intracranial aneurysms: symptomatic and asymptomatic. Clin Neurosurg 1974, 21:166-170.

19. Moyes PD. Surgical treatment of multiple aneurysms and of incidentally-discovered unruptured aneurysms. J Neurosurg 1971, 35:291-295.

20. Niakao S, Kikughi H, Takahashi N. Successful clipping of carotid-ophthalmic aneurysms through a contralateral pterional approach: report of two cases. J Neurosurg 1981, $54: 532-536$.

21 Nehls DC, Flom RA, Carter LP, Spetzler RF. Multiple intracranial aneurysms: determining the site of rupture. $J$ Neurosurg 1985, 63:342-348.

22. Ojemann RG. Management of the unruptured intracranial aneurysm. $N$ Engl Med 1981 , $304: 725-726$.

23. Oliveira E, Ribas GC, Yamashita M, Lourenzi M, Marino R Jr. Abordagem fronto-temporo-esfenoidal para a clipagem de aneurismas múltiplos localizados ipsi e contralateralmente. Apresentado no $\mathbf{1 6}^{\circ}$ Congresso Brasileiro de Neurocirurgia. Rio de Janeiro, agosto, 1986.

24. Paterson A, Bond MR. Treatment of multiple intracranial arterial aneurysms. Lancet 1973, $1: 1302-1304$.

25. Poppen JL, Fager CA. Multiple intracranial aneurysms. J Neurosurg 1959, 16:581-589.

26. Salazar JL. Surgical treatment of asymptomatic and incidental intracranial aneurysms. J Neurosurg 1980, 53:20-21.

27. Slamson DS, Hodosh RM, Clark WK. Surgical management of unruptured asymptomatic aneurysms. J Neurosurg 1977, 46:731-734.

28. Sekhar LN, Heros RC. Origin, growth and rupture of saccular aneurysms: a review. Neurosurgery 1981, 8:248-260.

29. Vadja J, Juhasz J, Pasztor E, Nyary I. Contralateral approach to bilateral and ophthalmic aneurysms. Neurosurgery 1988, 22:662-668.

30. Wiebers DO, Whisnant JP, Sundt T'M, O'Fallon WM. The significance of unruptured intracranial saccular aneurysms. J Neurosurg 1987, 66:23-29.

31. Wirth FP, Laws ER Jr, Piepgras D, Scott $R$. Surgical treatment of incidental intracranial aneurysms. Neurosurgery, 1983, 12:507-511.

32. Wood EH. Angiographic identification of the ruptured lesion in patients with multiple cerebral aneurysms. $J$ Neurosurg 1964, 21:182-198.

33. Yamada K, Hayakawa T, Oku Y, Maeda $Y$, Ushio $\mathbf{Y}$, Yoshimine $T$, Kawai R. Contralateral pterional approach for carotid-ophthalmic aneurysm: usefulness of high resolution metrizamide or blood computed tomognaphic cisternography. Neurosurgery 1984, 15:5-8.

34. Yasargil MG. Microsurgery Applied to Neurosurgery. Stuttgart: Georg Thieme, 1969. 